\title{
Cabozantinib S-malate
}

National Cancer Institute

\section{Source}

National Cancer Institute. Cabozantinib S-malate. NCI Thesaurus. Code C97938.

The s-malate salt form of cabozantinib, an orally bioavailable, small molecule receptor tyrosine kinase (RTK) inhibitor with potential antineoplastic activity. Cabozantinib strongly binds to and inhibits several RT Ks, which are often overexpressed in a variety of cancer cell types, including hepatocyte growth factor receptor (MET), RET (rearrang ed during transfection), vascular endothelial growth factor receptor types 1 (VEGFR-1), 2 (VEGFR2), and 3 (VEGFR-3), mast/stem cell growth factor (KIT), FMS-like tyrosine kinase 3 (FLT -

3), TIE-2 (TEK tyrosine kinase, endothelial), tropomyosin-related kinase B (TRKB) and AXL. This may result in an inhibition of both tumor growth and angiogenesis, and eventually lead to tumor regression. 